\title{
Bericht über die XVI. gesamtpolnische Wissenschaftskonferenz „Ihr Jugendlichen, Euch gehört die Zukunft (Parati Semper 1). In der Vorbereitung auf den Weltjugendtag", Tarnów, 24. Mai 2016
}

Am 24. Mai 2016 fand in der Aula Hl. Papst Johannes XXIII. im Gebäude des Priesterseminars in Tarnów die folgende und bereits XVI. gesamtpolnische Wissenschaftskonferenz „Ihr Jugendlichen, Euch gehört die Zukunft (Parati Semper 1). In der Vorbereitung auf den Weltjugendtag" statt, die von den Mitarbeitern des Lehrstuhls für Pädagogik und Katechese an der Theologischen Fakultät, Sektion Tarnów (WTST), der Päpstlichen Universität Johannes Paul II. in Krakau (UPJPII) zusammen mit den Mitarbeitern des Fachbereichs Polnische Philologie am Humanistischen Institut der Staatlichen Berufshochschule in Tarnów (PWSZ) organisiert wurde. Dies ist bereits die dritte Konferenz, welche die Dozenten der beiden Hochschulen gemeinsam initiiert und durchgeführt haben: Im Jahr 2015 begann die Zusammenarbeit mit den beiden internationalen Konferenzen „Pedagogy and Theology for the Future of Europe” (Krakau - Bibliothek UPJPII), sowie „The Family and Education in the Struggle for the Future of Europe" (Tarnów - Aula PWSZ). Die hier vorgestellte Konferenz stand unter der Schirmherrschaft von Seiner Magnifizenz, H. H. Prof. Dr. habil. Wojciech Zyzak (Rektor UPJPII) und Ihrer Magnifizenz, Prof. Dr. habil. Jadwiga Laska (Rektorin PWSZ). Teilnehmer waren Absolventen, Doktoranden und Studenten der WTST (Kleriker und Laienstudenten), Studenten der Fachbereiche Vorschulund Grundschulpädagogik mit dem Schwerpunkt Fremdsprachen der PWSZ, wie auch Lehrer und interessierte Besucher. 
Die Konferenz begann mit einem Gebet für die Organisatoren, die Jugendlichen und alle Pilger, die sich zwei Monate später anlässlich des Weltjugendtags in Krakau aufhalten sollten. H. H. Prof. Dr. habil. Józef Stala begrüßte die zum Gedankenaustausch Anwesenden im Namen des Vorbereitungsteams, insbesondere die Referenten sowie die Vertreter des Fachbereichs Polnische Philologie am Humanistischen Institut der Staatlichen Berufshochschule in Tarnów. Anschließend führte der Dekan der WTST, H. H. Dr. habil. Janusz Królikowski, Professor an der UPJPII, die Versammlung in die Konferenz ein, indem er auf Aktualität und Bedeutung der vorliegenden Thematik verwies. Er sprach den Organisatoren, allen voran dem Hauptorganisator Prof. J. Stala, seine Anerkennung für die außergewöhnlich effektive Zusammenarbeit zwischen den beiden Hochschulen aus.

Prof. Dr. habil. Józef Stala, Prorektor der UPJPII, eröffnete die Vortragsreihe mit seinem Referat „Światowe Dni Młodzieży - założenia, początki i rozwój” (Der Weltjugendtag - Gründung, Beginn und Entwicklung). Dabei erinnerte er an die ersten Bestrebungen und Anfänge des Weltjugendtags, bevor er dessen außerordentlich erfolgreiche Entwicklungsgeschichte beleuchtete. Unter der Bezugnahme auf die Worte Johannes Pauls II. versuchte der Referent folgende Frage zu beantworten: Was bedeutet der Weltjugendtag und wie ist er zu verstehen? Schlussfolgernd unterstrich er, dass der Weltjugendtag ein Aufbrechen zur Begegnung mit Gott darstelle, der durch Jesus Christus in die Geschichte der Menschheit eingetreten ist.

Das anschließende Referat „Światowe Dni Młodzieży w Krakowie na tle innych wydarzeń religijnych adresowanych do młodzieży” (Der Weltjugendtag in Krakau vor dem Hintergrund anderer religiöser Veranstaltungen für Jugendliche) trug Dr. habil. Elżbieta Bilska-Wodecka im Namen ihres größeren Teams vor, dem weiterhin folgende Mitglieder angehören: Dr. habil. Izabela Sołjan, Prof. Dr. habil. Antoni Jackowski, Justyna Liro (Magister), Maciej Trojnar (Magister), Edyta Kostrzewa (Jagiellonen-Universität in Krakau). Die Referentin verwies zunächst auf große Veranstaltungen, die wissenschaftlich untersucht wurden, und klärte dann die grundlegenden Begriffe: Festival und Happening. Darauf erläuterte sie die drei wesentlichen Elemente derartiger Veranstaltungen, die in der Attraktivität, der Visualisierung und der übermittelten Lehre bestehen. Dr. habil. E. Bilska-Wodecka ging auch auf die Organisation von bestimmten Versammlungszentren und Gemeinschaftserlebnissen während dieser Veranstaltungen ein. Abschließend betrachtete sie die Symbole des Weltjugendtags: das Kreuz, das Bild der Gottesmutter Maria sowie das Logo. 
Da der Weltjugendtag mit 5 bis 6 Tagen jedoch länger als andere typische Massenveranstaltungen dauere und eine intensive Werbung für die Stadt darstelle, sei er nach Ansicht der Referentin ein bedeutendes Medienereignis, aber ebenso eine attraktive Form, die Zeit mit Jugendlichen aus 160 bis 170 Ländern der Erde verbringen zu können. Dabei müsse bedacht werden, dass der Weltjugendtag als fröhliches Fest der Religion ein lebendiges Glaubenszeugnis geben solle, vor allem für Menschen, die noch auf der Suche sind. Der Vortrag befasste sich ebenfalls mit dem finanziellen Aspekt (Ausgaben und Gewinn) der bisherigen Weltjugendtage, bevor die nichtreligiösen Bereiche angesprochen wurden: 1. Werbung und Medien; 2. Direktes und indirektes Marketing; 3. Touristisches Potenzial.

Es folgte das Referat von Dr. habil. Elżbieta Osewska, wissenschaftlichdidaktische Mitarbeiterin und ordentliche Professorin an der Staatlichen Berufshochschule in Tarnów, mit dem Thema: „Nie lękajcie się Miłości, która stawia człowiekowi wymagania (PS 10)" (Habt keine Angst vor der Liebe, die dem Menschen bestimmte Forderungen stellt). Auf Grundlage der Daten, die von der Organisation Kirche in Not erhoben wurden, beschrieb sie die Situation von Christen in unterschiedlichen Regionen der Welt und ging dabei insbesondere auf Orte ein, wo das Bekenntnis zum christlichen Glauben zu Todesdrohungen führen kann oder zumindest den Verlust der gesellschaftlichen und beruflichen Position bedeutet. Im Hinblick auf die Situation in Polen stellte die Referentin die Frage, ob die jungen Menschen in Polen wohl den Mut hätten, ihren Glauben in ihrem Lebensumfeld zu bekennen - in der Familie, in der Schule, unter Gleichaltrigen? Dr. habil. E. Osewska nahm Bezug auf die Lehre von Papst Johannes Paul II. und zeigte damit die positiven Konsequenzen auf, die sich ergeben, wenn der Mensch die Liebe annimmt, die ihm von Jesus Christus angeboten wird.

Der nächste Referent, H. H. Dr. Roman Buchta (Schlesische Universität in Kattowitz) sprach zum Thema „Światowe Dni Młodzieży szansą na rozbudzenie świadomości chrzcielnej" (Der Weltjugendtag als Chance, das Bewusstsein für die Taufe auszubauen). Er erinnerte daran, dass die Taufe den Beginn des christlichen Lebens und die Aufnahme in die Gemeinschaft der Kirche darstelle, was von den jungen Menschen oft vergessen werde. Er betonte die Notwendigkeit, sowohl das Sakrament der Taufe als auch die Kirche so zu präsentieren, dass die Jugendlichen ihr Taufversprechen erneuern wollten und tagtäglich im Einklang mit empfangenen Taufe lebten. Der Weltjugendtag bedeute Freude, Dynamik und Austausch mit Gleichaltrigen, die sich zum selben Glauben bekennen. 
Somit werde er zur Chance, eine junge, lebendige und energievolle Kirche zu zeigen, der die Jugend gerne angehören wolle.

H. H. Dr. Andrzej Sułek (Päpstliche Universität Johannes Paul II. in Krakau) erörterte in seinem Vortrag „Obraz siebie młodych ludzi o różnym poziomie religijności" (Die Selbstwahrnehmung junger Menschen aufverschiedenen Niveaus der Religiosität) die Korrelation zwischen der Selbstwahrnehmung und der Religiosität. Es zeige sich - darauf verwies der Referent ausdrücklich - dass folgender Zusammenhang bestehe: je höher das Niveau der Religiosität, desto besser auch die gesellschaftliche Einordnung und Mitarbeit. Darüber hinaus führe das höchste Gebot, seinen Nächsten so wie sich selbst zu lieben, dazu, dass gesunde Selbstwahrnehmung und Selbstliebe ein Maß für die Nächstenliebe seien. Deshalb sollten bei einer gestörten Selbstwahrnehmung des jungen Menschen nicht nur pädagogische und psychologische sondern ebenso soziale und religiöse Aspekte betrachtet werden.

Dem abschließenden Referat von H. H. Dr. Waldemar Krzywiński (ErmlandMasuren-Universität in Olsztyna) „Doświadczenie piękna i prawdy jako droga młodych do spotkania z Bogiem" (Die Erfahrung des Schönen und der Wahrheit als Weg der Jugendlichen zur Begegnung mit Gott) lag die Überlegung zugrunde, dass die jungen Menschen in ihrem Leben nach Idealen und nach etwas suchen, das ihre Alltagserlebnisse übersteigt. Deshalb kann in einer Situation, in der sie sich auf verschiedene Art und Weise von Gott abwenden, die Erfahrung des Schönen und der Wahrheit zu einer Etappe werden, sich wieder auf den Weg zu Gott hin aufzumachen. Der Weltjugendtag zeige, dass in den Jugendlichen ein immenses Potenzial stecke. Es brauche jedoch Katecheten, Erzieher und Lehrer, die sie auf ihrem Weg der Suche begleiten, damit sie das Schöne und die Wahrheit finden und erfahren könnten.

Im Anschluss an die Vorträge stellten die Teilnehmer der Konferenz den Referenten Fragen und es entwickelte sich eine lebhafte Diskussion. Der Moderator, H. H. Prof. J. Stala, fasste die Ergebnisse der Konferenz zusammen und bestätigte noch einmal die Bedeutung sowie die Aktualität der aufgegriffenen und besprochenen Themen. Gleichzeitig verwies er darauf, dass noch vor dem Weltjugendtag in Krakau die Monographie „World Youth Days. A Testimony to the Hope of Young People" vom wissenschaftlichen Verlag der UPJPII erscheinen werde, an der unter der Redaktion von ihm selbst und A. Porębski eine Vielzahl von Autoren mitgewirkt haben. 

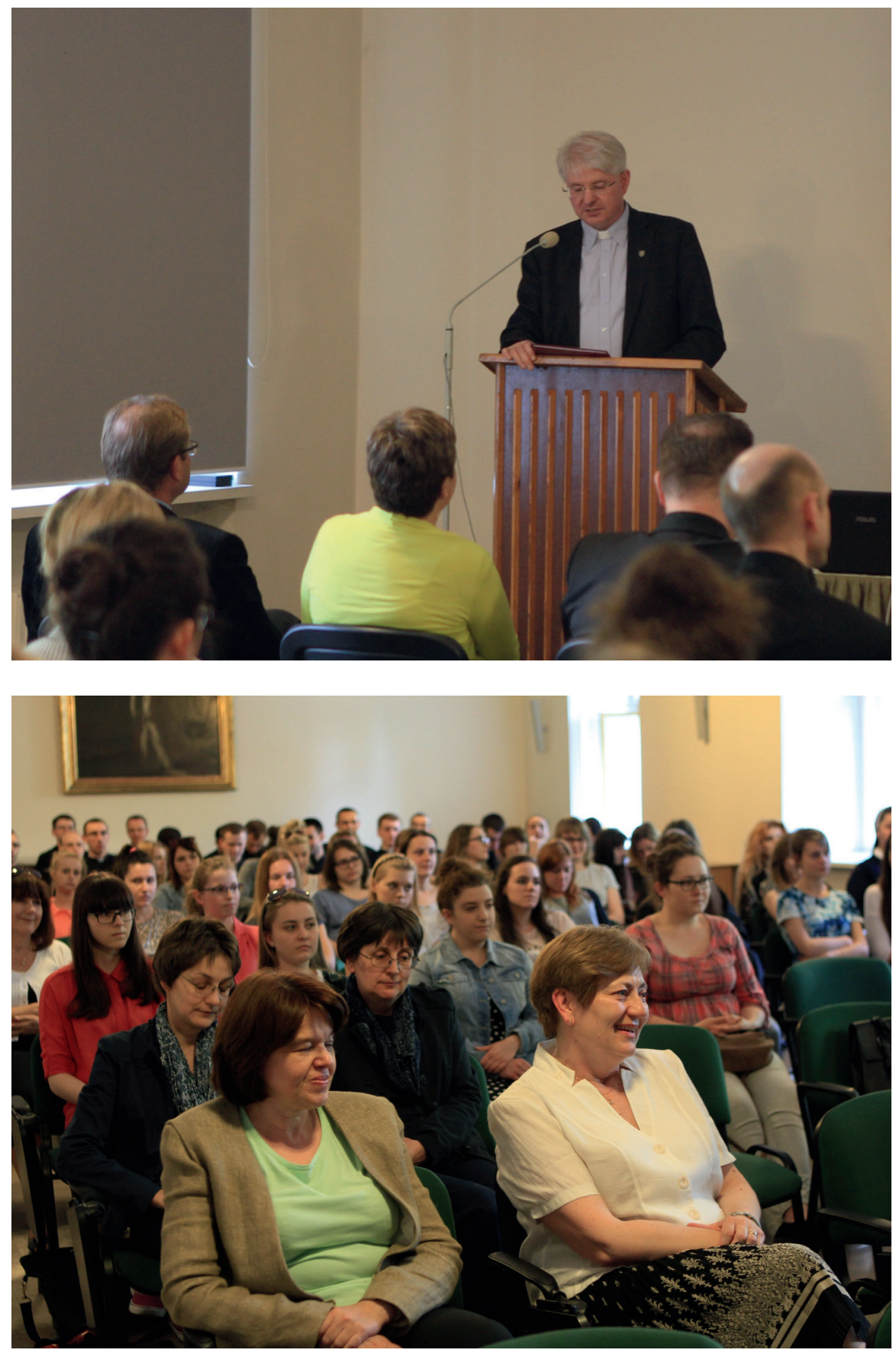